\title{
耐高温氧化铝气凝胶隔热复合材料研究进展
}

\author{
彭飞, 姜勇刚, 冯坚, 蔡华飞, 冯军宗, 李良军 \\ (国防科技大学 空天科学学院, 新型陶瓷纤维及其复合材料重点实验室, 长沙 410073)
}

\begin{abstract}
摘 要: 氧化铝气凝胶是一种高孔隙率、低密度、高比表面积、耐高温和低热导的纳米多孔材料, 在高温隔热领域(如 航天飞行器热防护系统、工业窑炉保温材料等)具有广阔的应用前景。但是, 纯氧化铝气凝胶因耐温性 (1000 ${ }^{\circ} \mathrm{C}$ 以 上)、力学性能和高温隔热性能相对较差难以直接应用, 需要引入增强相和遮光组分制备成气凝胶复合材料以进行 改善。本文对耐高温氧化铝气凝胶的制备、氧化铝气凝胶隔热复合材料的制备及性能等方面的最新研究进展进行 了综述。研究人员通过原位掺杂改性、沉积改性、有机链和炭涂层改性等方法提高了氧化铝气凝胶的热稳定性。 在氧化铝气凝胶中引入晶须、颗粒、多孔骨架和纤维等增强相, 能够大幅提高其力学性能; 纤维和遮光剂的协同作 用, 能够提高氧化铝气凝胶抑制红外辐射的能力, 显著降低高温热导率。本文还提出了后续的研究方向: 对氧化铝 气凝胶的密度、微观结构进行精细调控, 再引入合适的异质元素和遮光剂, 以进一步提高气凝胶的热稳定性和复合 材料的隔热性能; 深入研究复合材料在高温下结构和性能的演化, 以及氧化铝气凝胶和增强相之间的相互作用。作 为一种新型的隔热材料, 氧化铝气凝胶复合材料将在高温隔热领域发挥其优势并逐步实现广泛应用。
\end{abstract}

关 键 词: 氧化铝气凝胶; 隔热; 复合材料; 增强相; 遮光剂; 综述

中图分类号: TB332 文献标志码: A

\section{Research Progress on Alumina Aerogel Composites for High-temperature Thermal Insulation}

\author{
PENG Fei, JIANG Yonggang, FENG Jian, CAI Huafei, FENG Junzong, LI Liangjun
}

(Science and Technology on Advanced Ceramic Fibers and Composites Laboratory, College of Aerospace Science and Technology, National University of Defense Technology, Changsha 410073, China)

\begin{abstract}
As a nano-porous material with high porosity, low density, high specific surface area, excellent heat-resistance, and low thermal conductivity, the alumina aerogel shows broad application prospect in high-temperature thermal insulating areas such as the thermal protective system for space vehicles and the thermal insulation for industrial kilns. However, pristine alumina aerogels cannot be directly used because of their relatively poor thermal resistance above $1000{ }^{\circ} \mathrm{C}$, mechanical strengths and high-temperature thermal insulating performance. They need to be improved by introducing reinforcements and opacifiers (namely the alumina aerogel composite). This paper summarizes the latest research progress of synthesis of heat-resistant alumina aerogels, and preparation and properties of alumina aerogel composites. Researchers improved the thermal stability of alumina aerogels by modifications such as in-situ doping, deposition, organic chains or carbon coatings. The introduction of whiskers, particles, porous skeletons or fibers into alumina aerogels contributes to considerable improvement of mechanical performance. The cooperation of fibers and opacifiers helps to increase the inhibition of infrared radiation and lower the high-temperature thermal conductivity of alumina aerogels. The future of alumina aerogel composites is also proposed: a) finely tailoring the bulk density, micro structures, and introducing proper foreign elements and opacifiers, are supposed to further optimize the thermal stability of alumina aerogels and thermal insulating performance of their composites; b) deeper attention should also be paid on the evolution of structure and properties of composites at elevated temperatures, and on the interaction between alumina aerogel and reinforcement. As a novel thermal insulation, alumina aerogel composites are expected to take their advantages and be widely applied in the future.
\end{abstract}

Key words: alumina aerogel; thermal insulation; composite; reinforcement; opacifier; review

收稿日期: 2020-07-20; 收到修改稿日期：2020-09-17; 网络出版日期：2020-10-30

基金项目: 湖南省自然科学基金面上项目(2018JJ2469) Hunan Provincial Natural Science Foundation (2018JJ2469)

作者简介: 彭飞(1985-), 男, 博士研究生. E-mail: feijigong@126.com PENG Fei (1985-), male, PhD candidate. E-mail: feijigong@126.com

通信作者: 姜勇刚, 副研究员. E-mail: jygemail@nudt.edu.cn; 冯 坚, 研究员. E-mail: fengj@nudt.edu.cn JIANG Yonggang, associate professor. E-mail: jygemail@nudt.edu.cn; FENG Jian, professor. E-mail: fengj@nudt.edu.cn 
随着航天飞行器不断向高马赫数和长航时的方 向发展, 飞行器表面的气动加热环境更为恶劣 ${ }^{[1]}$, 对热防护系统的防隔热性能提出了极为苛刻的要求, 亟需轻质、耐更高温度 $\left(1000{ }^{\circ} \mathrm{C}\right.$ 以上 $)$ 、隔热性能更 优异且具有较好力学性能的新型材料。在民用领域, 工业窑炉(水泥回转窑、钢铁高炉/转炉、陶瓷窑等, 工作温度 $200 \sim 2500{ }^{\circ} \mathrm{C}$ ) 在总的能源消耗中占据很大 比重, 采用传统的耐火砖、陶瓷纤维等作为保温材 料, 热导率偏高, 造成了较高的能量耗散, 需要采 用新型的高效隔热材料降低能源消耗。

气凝胶是一种由纳米尺度颗粒组成的高孔隙率 和低密度材料, 其复杂的三维网络结构、纳米尺度 的孔径显著抑制了固态和气态热传导, 因而具有 极低的热导率 ${ }^{[2]}$ 。陶瓷气凝胶 (如氧化硅 ${ }^{[3-4]}$ 、氧化 铝 ${ }^{[5-7]}$ 、氧化锆 ${ }^{[8]}$ 、碳化硅 ${ }^{[9]}$ 、氮化硼 ${ }^{[10]}$ 等气凝胶) 因具有良好的耐高温和隔热性能而受到广泛关注。 氧化硅气凝胶是目前研究最为成熟的气凝胶材料, 已经作为高性能隔热材料得到了广泛应用 ${ }^{[2]}$, 但是 氧化硅气凝胶在高温下会发生孔结构的破坏，其短 期使用温度上限一般不超过 $800{ }^{\circ} \mathrm{C}^{[11-12]}$ 。相比于氧 化硅气凝胶, 氧化铝气凝胶耐温性更高, 在 $1000{ }^{\circ} \mathrm{C}$ 时仍然能够保持纳米孔结构 ${ }^{[5]}$, 并具有很低的热导 率 $\left(30{ }^{\circ} \mathrm{C}\right.$ 时为 $0.029 \mathrm{~W} /(\mathrm{m} \cdot \mathrm{K})$ ), 是目前有氧环境下耐 温和隔热性能突出的气凝胶材料, 在航空航天、工 业窑炉等温度更高的隔热领域具有很大的应用潜 力。但是, 纯氧化铝气凝胶存在耐温性有待提升(最 高使用温度一般不超过 $1000{ }^{\circ} \mathrm{C}$ )、力学性能 ${ }^{[5,13]}$ 和 高温隔热性能较差(对红外辐射的透过性很高, 在 高温下热导率急剧上升 ${ }^{[5,14-15]}$ ) 的问题, 这是阻碍其 在高温隔热领域实际应用的主要瓶颈。

自 2008 年以来, 研究人员先后发展了多种耐高 温氧化铝气凝胶, 并在氧化铝气凝胶中引入力学增 强相和红外遮光剂, 在提高氧化铝气凝胶耐温性、 力学性能和高温隔热性能三方面开展了大量的研究 工作, 本文对近年来这三方面的研究工作进行了详 细总结, 对存在的问题进行了分析, 并对后续的研 究方向提出了展望。

\section{1 耐高温氧化铝气凝胶制备}

如图 1 所示, 氧化铝气凝胶的制备过程一般包 括溶胶-凝胶、老化、溶剂置换和干燥四个环节 ${ }^{[16-17]}$ 。 用于制备氧化铝溶胶的先驱体主要包括铝醇 盐 ${ }^{[5-6,18-19]}$ 、无机铝盐 ${ }^{[13,20-22]}$ 、勃姆石粉 ${ }^{[20]}$ 或勃姆石 溶胶 ${ }^{[23-24]}$ 。铝醇盐或无机铝盐通过水解反应形成具 有 $\mathrm{Al}-\mathrm{OH}$ 的中间体，中间体通过 $\mathrm{Al}-\mathrm{OH}$ 的缩合反 应生成含 $\mathrm{Al}-\mathrm{O}-\mathrm{Al}$ 的溶胶颗粒, 颗粒间发生交联和
团簇, 进一步生成三维网络结构并形成凝胶。铝醇 盐活性很高，极易水解和缩聚，制备过程难以控制, 通常需要加入螯合剂(乙酰乙酸乙酯、乙酰丙酮等 ${ }^{[6,18]}$ ) 形成具有 $\mathrm{Al}-\mathrm{O}-\mathrm{C}$ 的螯合物以降低其反应活性; 无 机铝盐在水溶液中以水合离子形式存在, 通过环氧 化物的开环反应, 消耗氢离子使 $\mathrm{pH}$ 缓慢上升, 生成 $\mathrm{Al}-\mathrm{OH}$ 并逐步发生缩聚反应形成三维网络结构 ${ }^{[13]}$; 勃姆石溶胶则是通过颗粒的絮凝作用形成凝胶 ${ }^{[24]}$ 。 在老化过程中, 凝胶网络结构的完整性和强度进一 步提高; 凝胶孔隙内的水和其它副产物需要通过溶 剂置换进行清除。为了避免凝胶孔内溶剂在干燥过 程中产生毛细管力引起气凝胶的收缩、开裂和孔结 构的破坏, 通常采用超临界态的乙醇 ${ }^{[14,25-29]}$ 或二 氧化碳 ${ }^{[13,30]}$ 作为介质实现凝胶的干燥。相对于超临 界干燥工艺, 冷冻干燥和常压干燥工艺制备的气凝胶 收缩更大, 但成本更低, 也获得了关注和研究 ${ }^{[31-33]}$, 通常将这两种干燥方式得到的产物分别称为氧化铝 冻凝胶和干凝胶。

氧化铝气凝胶一般为拟薄水铝石或者勃姆石晶 相，随着温度升高会逐渐发生脱水 $\rightarrow$ 过渡相氧化铝 $\rightarrow$ $\alpha$ 相氧化铝的转变过程。构成氧化铝气凝胶三维网 络结构的颗粒一般为纳米尺度，具有很高的活性， 在 $1000{ }^{\circ} \mathrm{C}$ 以上开始发生显著的颗粒烧结和相变 ${ }^{[6-7,24]}$, 并伴随着体积收缩和孔结构坞塌, 导致比表面积和 性能下降。为了进一步提高氧化铝气凝胶的热稳定 性，研究人员采用元素掺杂、沉积改性等方法抑制 氧化铝气凝胶的烧结和 $\alpha$ 相转变, 取得了较大进展。

\section{1 原位掺杂改性}

在氧化铝气凝胶中引入硅元素是提高其耐温性 的常用方法 ${ }^{[7,18,34-39]}$ 。Horiuchi 等 ${ }^{[34]}$ 通过添加(2.5 $10) \mathrm{wt} \%$ 的氧化硅, 使氧化铝气凝胶的 $\alpha$ 相转变温度 从 $1100{ }^{\circ} \mathrm{C}$ 升高至 $1400{ }^{\circ} \mathrm{C}$ 。均匀分布在氧化铝气凝 胶网络结构中的硅，抑制了高温下铝离子的表面扩 散, 从而抑制了颗粒的烧结和 $\alpha$ 相转变, 经 $1300{ }^{\circ} \mathrm{C}$ 处理 $5 \mathrm{~h}$ 后比表面积仍有 $80 \mathrm{~m}^{2} / \mathrm{g}$ 。Osaki 等 ${ }^{[31]}$ 观察 到添加 $10 \mathrm{wt} \%$ 氧化硅的氧化铝冻凝胶 (冷冻干燥) 经 $1200{ }^{\circ} \mathrm{C}$ 热处理 $5 \mathrm{~h}$ 后, 仍然以 $\gamma-\mathrm{Al}_{2} \mathrm{O}_{3}$ 相为主, 并认 为热稳定性提高是由于硅离子占据了 $\gamma-\mathrm{Al}_{2} \mathrm{O}_{3}$ 的四 面体空位使总的空位数量减少，从而抑制了高温下 的晶格振动和 $\alpha$ 相转变。冯坚等 ${ }^{[25]}$ 发现当氧化铝氧化硅气凝胶中硅含量为(6.1 13.1) wt \% 时, 硅原子 能够完全填充氧化铝的四面体空位, 此时其热稳定 性明显高于纯氧化铝气凝胶, $1200{ }^{\circ} \mathrm{C}$ 时的比表面积 为 97 116 $\mathrm{m}^{2} / \mathrm{g}$ 。 Pakharukova 等 ${ }^{[18]}$ 则提出, 氧化硅 的引入增加了氧化铝气凝胶的各向异性程度, 减少 了颗粒间颈部接触的机率，从而抑制了 $\alpha-\mathrm{Al}_{2} \mathrm{O}_{3}$ 晶 相的形核和晶粒长大。Peng 等 ${ }^{[39]}$ 发现含适量硅的氧 


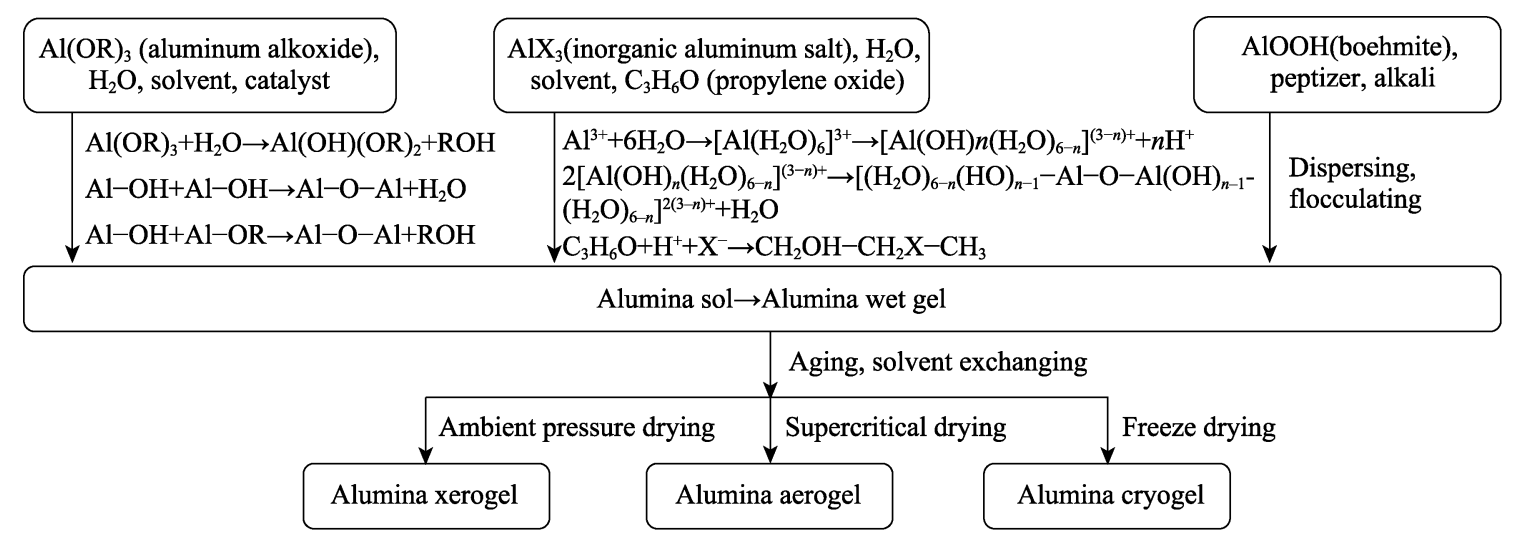

图 1 氧化铝气凝胶的制备过程 $(\mathrm{R}$ 代表烷氧基, $\mathrm{X}$ 代表氯离子等阴离子)

Fig. 1 Preparation of alumina aerogels ( $\mathrm{R}$ refers to the alkoxyl group and $\mathrm{X}$ refers to the anion such as $\mathrm{Cl}^{-}$)

化铝-氧化硅气凝胶的热稳定性显著高于纯氧化 铝气凝胶(图 2(a, b ) ), 均匀分布在氧化铝气凝胶 中的硅(图 2(c))抑制了铝原子的表面扩散和重排, 在高温下转化为莫来石相，消耗了一部分可能发生 $\alpha$ 相转变的氧化铝，同时减少了氧化铝颗粒间的颈 部接触，最终抑制了 $\alpha$ 相转变。

除了掺杂硅元素, 在氧化铝气凝胶中添加碱土 金属、稀土元素等也能够提高其热稳定性。研究者 采用无机铝盐为先驱体、环氧丙烷为促凝剂制备了 镂 ${ }^{[40-42]}$ 、钎 ${ }^{[43-44]}$ 、锶 ${ }^{[45]}$ 、铅 ${ }^{[46]}$ 等元素掺杂的氧化铝 气凝胶。Tokudome 等 ${ }^{[40]}$ 制备的氧化铝-氧化镧气凝 胶经较长时间高温处理 $\left(1100{ }^{\circ} \mathrm{C}, 24 \mathrm{~h}\right)$ 后, 仍能保 持较高的比表面积 $\left(82.5 \mathrm{~m}^{2} / \mathrm{g}\right)$, 热稳定性的提高主 要归结于生成了锞铝化合物。周洁洁等 ${ }^{[4]}$ 通过在氧 化铝气凝胶中添加钎, 使其在 $1000{ }^{\circ} \mathrm{C}$ 时保持完整 的纤维状网络结构和高的比表面积 $\left(410 \mathrm{~m}^{2} / \mathrm{g}\right)$, $1200{ }^{\circ} \mathrm{C}$ 时仍保持过渡相 $\left(\delta-\mathrm{Al}_{2} \mathrm{O}_{3}\right)$, 并在高温下氧化
钎和氧化铝形成了固溶体, 降低了铝离子的扩散速 率，从而抑制了气凝胶的烧结和相变。孙雪峰等 ${ }^{[45]}$ 发现添加适量锶的氧化铝气凝胶经 $1200{ }^{\circ} \mathrm{C}$ 处理后 的比表面积达到 $122 \mathrm{~m}^{2} / \mathrm{g}$, 认为热稳定性的提高是 由于 $\mathrm{SrO}$ 抑制了气凝胶的烧结，而高温下生成的 $\mathrm{SrO} \cdot 6 \mathrm{Al}_{2} \mathrm{O}_{3}$ 抑制了 $\alpha$ 相转变。

\section{2 沉积改性}

$\mathrm{Zu}$ 等 ${ }^{[47]}$ 采用仲丁醇铝(ASB) 和正硅酸乙酯 (TEOS)溶胶多次浸泡氧化铝凝胶, 借助化学沉积方 式(图 3(a))使凝胶颗粒增大、网络结构增强，并在 表面生成氧化硅颗粒，显著提高了氧化铝气凝胶 抵抗烧结和相转变的能力, 经 $1300{ }^{\circ} \mathrm{C}$ 处理 $2 \mathrm{~h}$ 后 线性收缩仅为 $4 \%$ (图 3(b)), 比表面积为 $139 \mathrm{~m}^{2} / \mathrm{g}$, 仍保持为片叶状(图 3(c))。利用相似的原理, $\mathrm{Zu}$ 等 ${ }^{[7]}$ 以 ASB 和 TEOS 溶胶作为改性液，在超临界干燥过 程中实现改性，增大了氧化铝气凝胶颗粒的尺寸和 骨架强度, 减少了颗粒间的颈部接触点; 进一步采

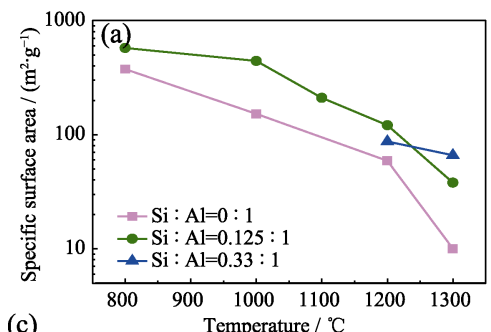

(c)

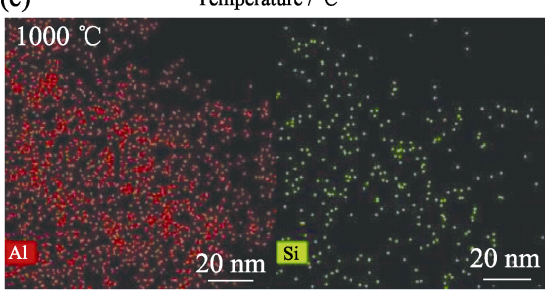

$\mathrm{Si}: \mathrm{Al}=0.125: 1$

(b)
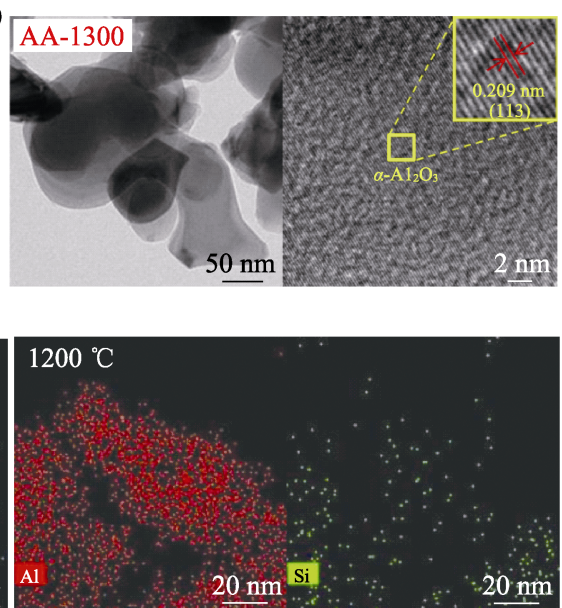

$\mathrm{Si}: \mathrm{Al}=0.091: 1$
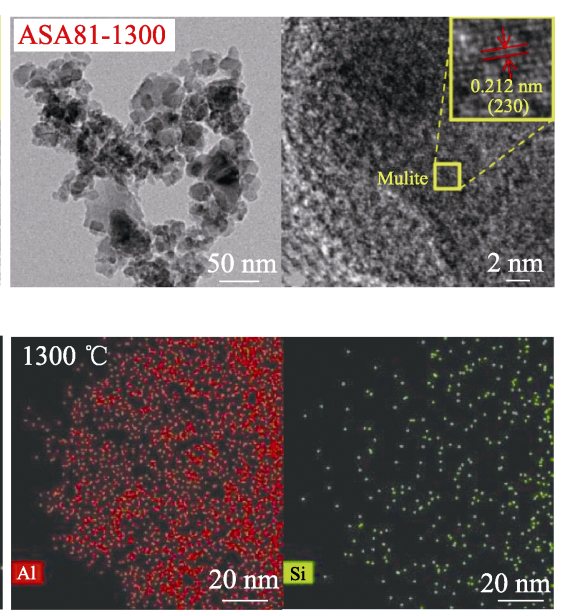

$\mathrm{Si}: \mathrm{Al}=0.11: 1$

图 2 高温热处理后的氧化铝-氧化硅气凝胶 ${ }^{[39]}$

Fig. 2 Alumina-silica aerogels after heat-treatment ${ }^{[39]}$

(a) Specific surface areas of aerogels with different molar ratios of Si; (b) TEM photos of the pristine alumina aerogel (AA-1300) and alumina-silica aerogel calcined at $1300{ }^{\circ} \mathrm{C}$ (ASA81-1300); (c) Distributions and atomic ratios of Si and Al in the aerogel with a theoretical Si/Al molar ratio of $0.125: 1$ (Bars representing $20 \mathrm{~nm}$; the red and green color representing Si and Al, respectively) 


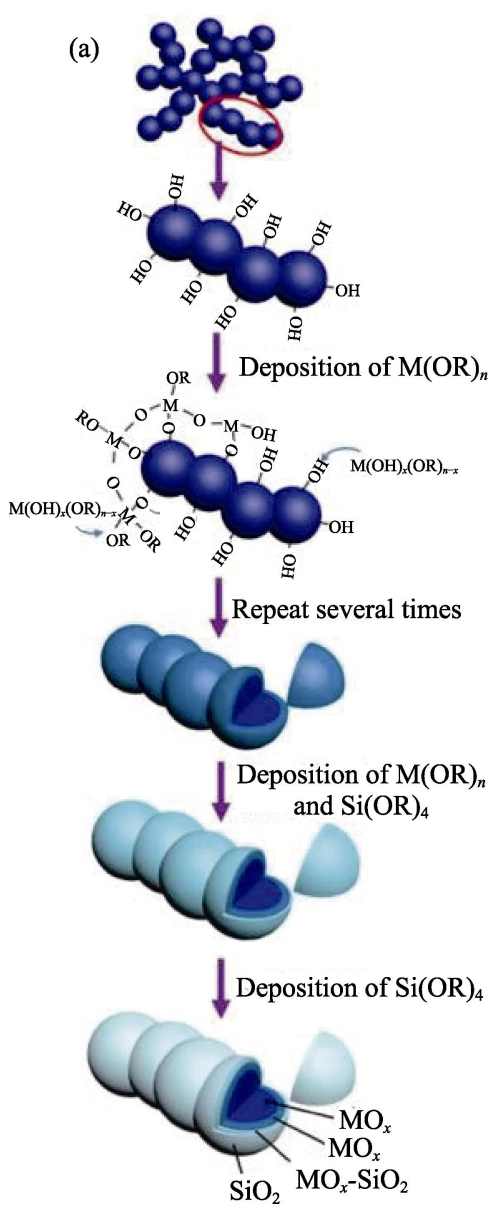

(b)

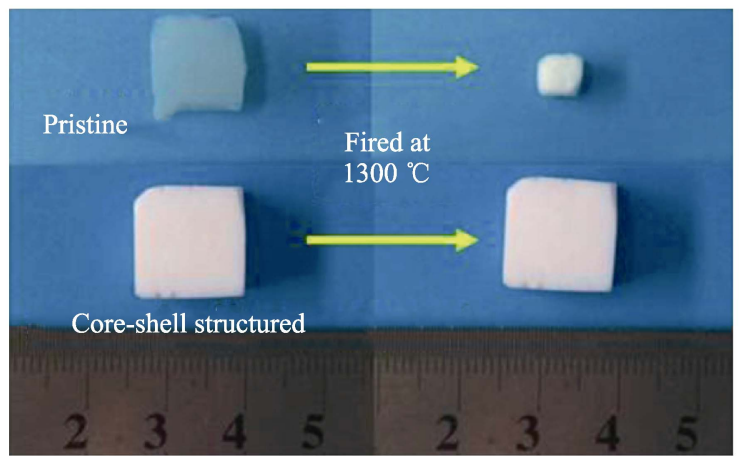

(c)
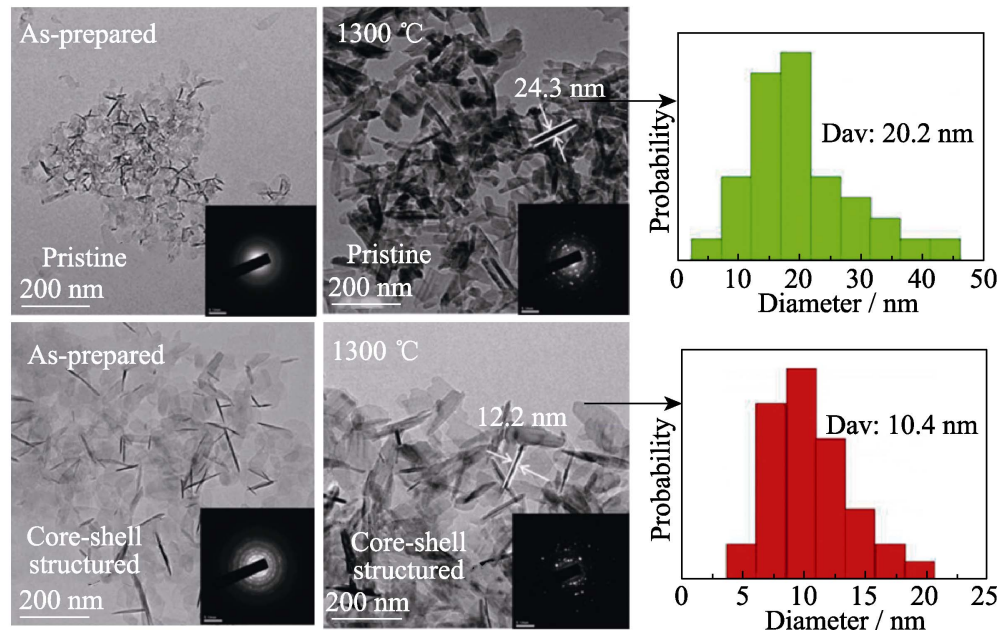

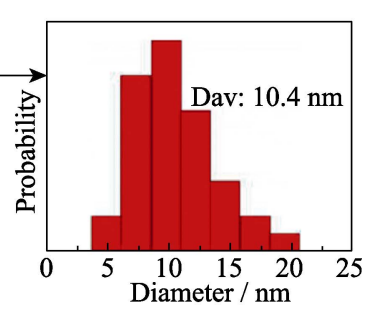

图 3 硅改性氧化铝气凝胶 ${ }^{[47]}$

Fig. 3 Si-modified alumina aerogels ${ }^{[47]}$

(a) Deposition modification of the gels; (b) Macro and (c) Micro changing of pristine and Si-modified alumina aerogels after heat-treatment at $1300{ }^{\circ} \mathrm{C}$

用六甲基二硅氮烷与气凝胶颗粒表面反应引入 $\mathrm{Si}-\mathrm{CH}_{3}$ 基团在高温下转变成 $\mathrm{SiO}_{2}$ 颗粒, 能够抑制气 凝胶的烧结和相转变。制备的改性氧化铝气凝胶经 $1300{ }^{\circ} \mathrm{C}$ 处理 $2 \mathrm{~h}$ 后, 线性收缩仅为 $5 \%$, 比表面积高 达 $125 \mathrm{~m}^{2} / \mathrm{g}$ 。

\section{3 其它方法}

Mizushima 等 ${ }^{[48]}$ 通过在氧化铝气凝胶颗粒表面 引入有机链, 替换原有的 $\mathrm{Al}-\mathrm{OH}$, 抑制了颗粒表面 $\mathrm{Al}-\mathrm{OH}$ 的缩合和 $\alpha$ 相转变。经 $1200{ }^{\circ} \mathrm{C}$ 处理 $5 \mathrm{~h}$ 后, 氧 化铝气凝胶的微观形貌仍然保持针状, 比表面积接 近 $100 \mathrm{~m}^{2} / \mathrm{g}$, 未发生 $\alpha$ 相转变, 表明其热稳定性得 到了改善。Yakovleva 等 ${ }^{[49]}$ 制备了类似于核一壳结构 的炭涂层氧化铝气凝胶, 其 $\alpha$ 相转变温度以及经高 温热处理(1100 1500 ${ }^{\circ} \mathrm{C}$ 氩气气氛)后的比表面积显 著高于未涂层的氧化铝气凝胶。氧化铝颗粒外部 的耐高温惰性壳层(炭)产生了隔离效应, 大大减 少了颗粒间的接触, 从而显著抑制了颗粒的烧结 和相变。

在溶胶制备过程中加入异质元素先驱体, 可获 得掺杂元素分布均匀的氧化铝气凝胶。通过调控铝
源和异质元素先驱体的比例, 能够较为精确地设计 元素掺杂量。适量、均匀的掺杂元素能够有效地抑 制氧化铝的烧结和相变, 提高其热稳定性, 其中掺 杂硅元素的改善效果最为显著。通过沉积改性方法 增强氧化铝凝胶的骨架及引入氧化硅颗粒, 能够显 著提高氧化铝气凝胶的耐温性, 但是在制备过程中 通常需要进行多次溶剂置换和改性, 并消耗大量的 改性液，其制备工艺的简易性和成本方面仍有待进 一步优化。有机链改性方法对氧化铝气凝胶热稳 定性的提高比较有限; 炭隔离相的方法是在氧化铝 气凝胶网络结构中引入炭涂层, 其在高温有氧条件 下会发生氧化，丧失其隔离相的作用。因此，在气凝 胶中原位掺杂硅元素是目前较好的制备耐高温氧化 铝气凝胶的途径。

\section{2 氧化铝气凝胶复合材料的制备及力 学性能}

氧化铝气凝胶的内部结构以孔隙为主 ${ }^{[5]}$ (图 4(a)), 其典型密度(不高于 $0.2 \mathrm{~g} / \mathrm{cm}^{3}$ )和弹性模量(图 4(b)) 

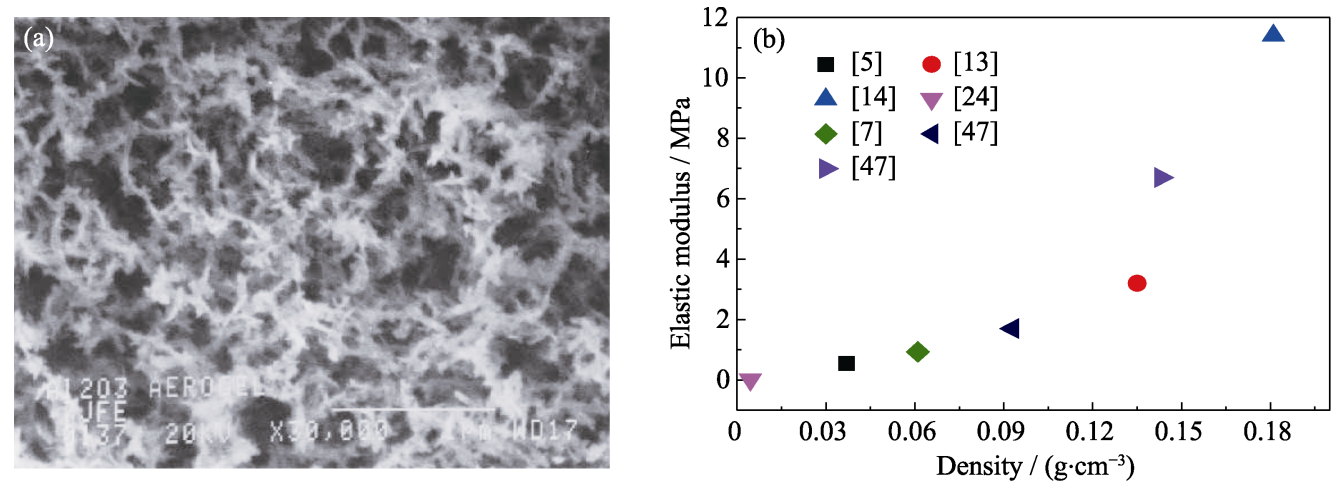

图 4 典型氧化铝气凝胶的(a)多孔结构 ${ }^{[5]}$ 和(b)弹性模量

Fig. 4 (a) Porous structure ${ }^{[5]}$ and (b) elastic moduli of alumina aerogels

Whisker or particle reinforced

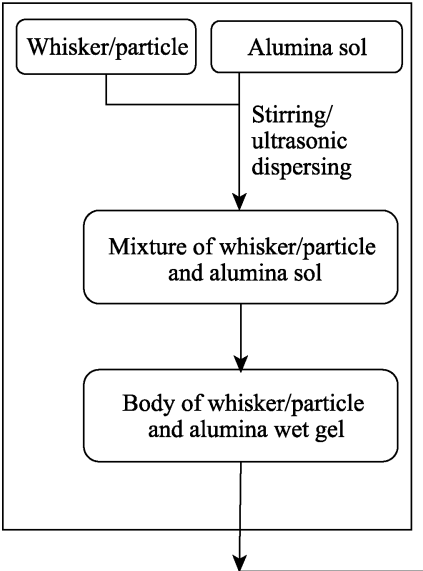

Porous skeleton reinforced

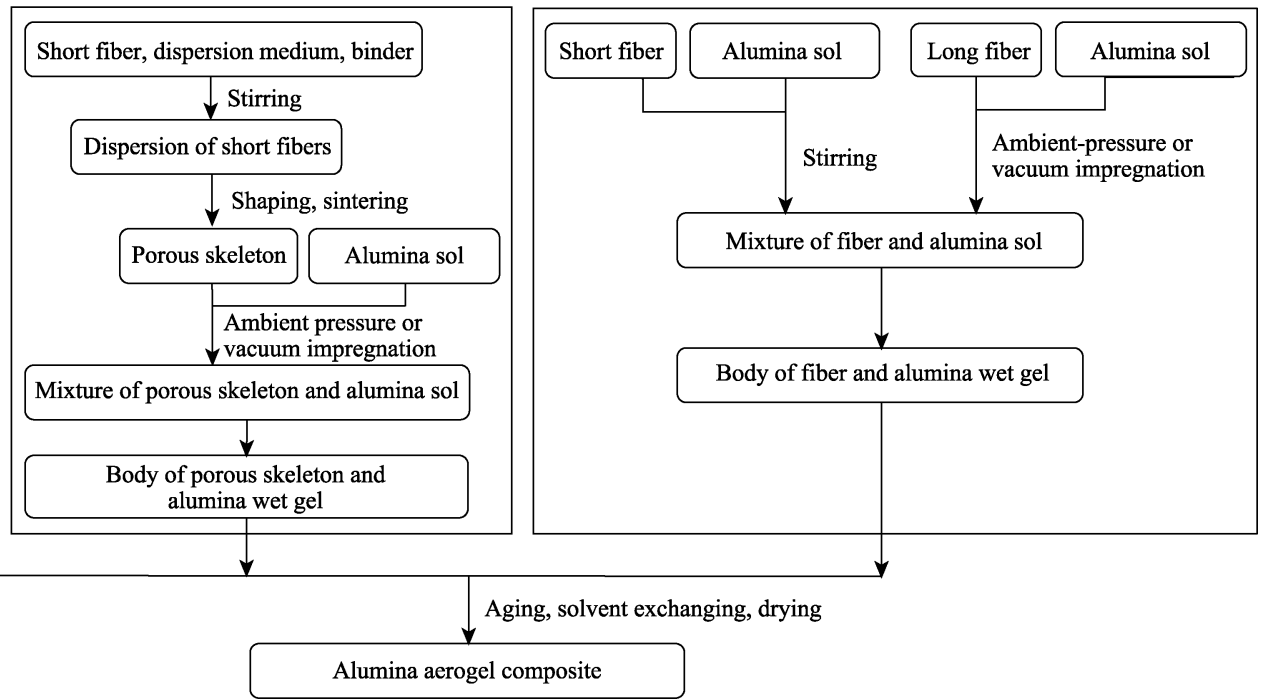

图 5 氧化铝气凝胶复合材料的制备方法

Fig. 5 Synthesis of alumina aerogel composites

远低于致密氧化铝材料，并且氧化铝本身是一种脆 性材料, 导致氧化铝气凝胶的骨架强度很低, 容易 被破坏。Baumann 等 ${ }^{[13]}$ 观察到氧化铝气凝胶脆性很 大，易碎裂，可操作性较差。

为了改善纯氧化铝气凝胶的力学性能, 研究者 通常需要引入力学增强相制备氧化铝气凝胶复合材 料，常见的增强相包括晶须、颗粒或纤维等一类强 韧的材料。由图 5 可见, 氧化铝气凝胶复合材料的 制备过程主要涉及到液(氧化铝溶胶)固(增强相)两 相的复合，增强相的种类不同，其与氧化铝气凝胶 的复合方式也有较大差异。

\section{1 晶须或颗粒增强}

由于晶须或颗粒的尺寸较小, 研究人员通常采 用机械搅拌、超声振动等手段将其分散在氧化铝溶 胶中, 进一步制备出氧化铝气凝胶复合材料。 Mizushima 等 ${ }^{[50]}$ 通过加入乙酰乙酸乙酯(螯合剂)调 控氧化铝溶胶的活性, 在高速搅拌和超声分散条件 下使碳化硅晶须分散在溶胶中, 采用超临界干燥制
备了碳化硅晶须增强氧化铝气凝胶复合材料。Hou 等 ${ }^{[27]}$ 将嗍酸铝晶须加入氧化铝-氧化硅溶胶中搅拌 后, 通过升温使其快速形成凝胶, 再经超临界干燥 制备了氧化铝-氧化硅气凝胶复合材料(图 6(a,b))。 在压缩载荷下, 复合材料中的晶须通过桥连和拔出 机制消耗能量, 因此具有较高的压缩强度(图 6(c))。 隋超 ${ }^{[51]}$ 通过引入纤维素晶须改善了氧化铝气凝胶 的力学性能, 当纤维素含量较高时, 复合材料具有 明显的柔性。曹风朝 ${ }^{[52]}$ 将凹凸棒土颗粒进行预分散 形成悬浮液后再加入氧化铝溶胶中, 获得了颗粒增 强氧化铝气凝胶复合材料, 复合材料的压缩强度很 高(74.5 MPa)，但密度偏大 $\left(0.8 \mathrm{~g} / \mathrm{cm}^{3}\right)$ 。

由于重力作用, 晶须或颗粒在氧化铝溶胶中容 易产生沉降而导致制备的复合材料不均匀, 因此, 掌握合适的添加时机和工艺参数是制备过程的关键。

\section{2 多孔骨架增强}

引入增强相的另外一种方式, 是将陶瓷纤维制 备成本身具有一定强度的多孔骨架再浸渍氧化铝溶 
胶。用于制备多孔骨架的纤维主要包括莫来石、氧 化锆和石英纤维等。

孙晶晶等 ${ }^{[53]}$ 以莫来石纤维为原料, 经高温烧结 得到多孔骨架(隔热瓦), 再浸渍氧化铝溶胶, 采用 乙醇超临界干燥制备得到常温热导率(热流计法) 为 $0.058 \mathrm{~W} /(\mathrm{m} \cdot \mathrm{K})$ 的隔热瓦复合氧化铝气凝胶材料, 其 压缩和拉伸强度分别达到 $1.48 、 0.58 \mathrm{MPa}$, 经 $1400{ }^{\circ} \mathrm{C}$ 处理 $0.5 \mathrm{~h}$ 后厚度收缩率为 $2 \%$ 。

Zhang 等 ${ }^{[37]}$ 则采用氧化锆纤维为原料, 经过挤 压成型和高温烧结得到高孔隙率(>91\%)的多孔骨 架(图 7(a)), 再将氧化铝-氧化硅溶胶浸渍于多孔骨 架中, 制备得到复合材料(图 7(b))。由图 7(c) 可知, 多孔骨架本身具有较好的力学性能, 与氧化铝-氧
化硅气凝胶复合后, 密度稍有增大, 压缩强度 (1.22 MPa)大幅提高。

$\mathrm{Yu}$ 等 ${ }^{[33]}$ 通过短切石英纤维和粘接剂共同烧结 得到多孔骨架(图 8(a)), 再浸渍氧化铝-氧化硅溶胶, 常压干燥后得到复合材料。粘接剂将纤维结合在一 起(图 8(b, c)), 形成具有一定抗压强度 $(0.3 \mathrm{MPa})$ 的 多孔骨架，因而复合材料具有较高的压缩强度 $(10 \%$ 应变, $0.85 \mathrm{MPa}$ )。

Zou 等 ${ }^{[54]}$ 采用冷冻干燥和烧结工艺得到莫来石 纤维多孔骨架, 再浸渍氧化铝-氧化硅溶胶, 经超临 界干燥后得到密度为 $0.227 \sim 0.304 \mathrm{~g} / \mathrm{cm}^{3}$ 的气凝胶复 合材料。复合材料的压缩应力 ( $3 \%$ 应变)和杨氏模量 分别为 $0.02 \sim 0.11 \mathrm{MPa} 、 0.78 \sim 3.58 \mathrm{MPa}$, 相比莫来石
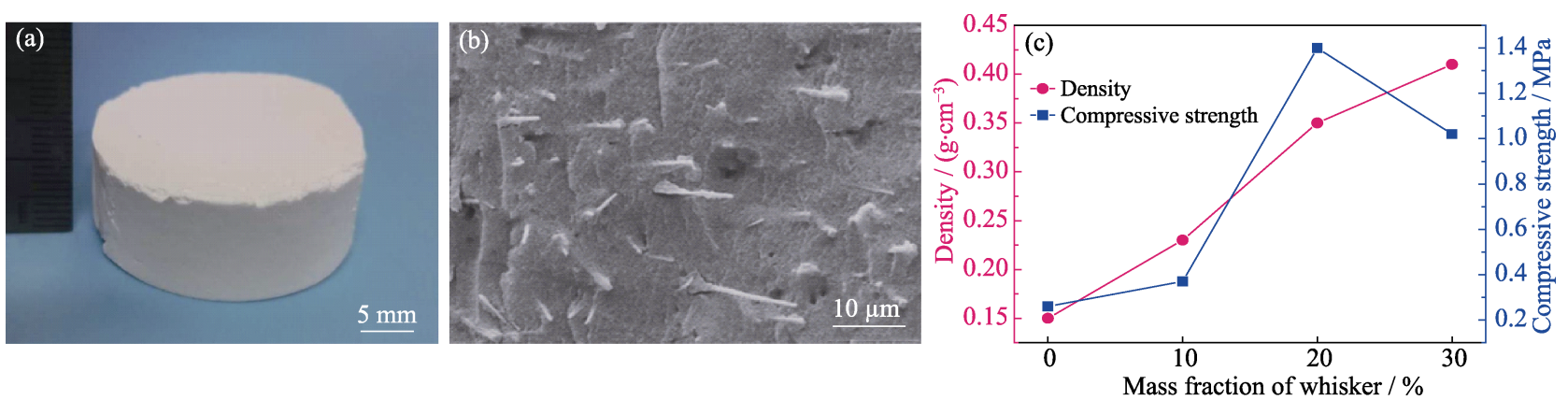

图 6 硼酸铝晶须增强氧化铝-氧化硅气凝胶复合材料 ${ }^{[27]}$

Fig. 6 The aluminum borate whisker reinforced alumina-silica aerogel composite ${ }^{[27]}$

(a) Macro and (b) micro morphology; (c) Mechanical properties
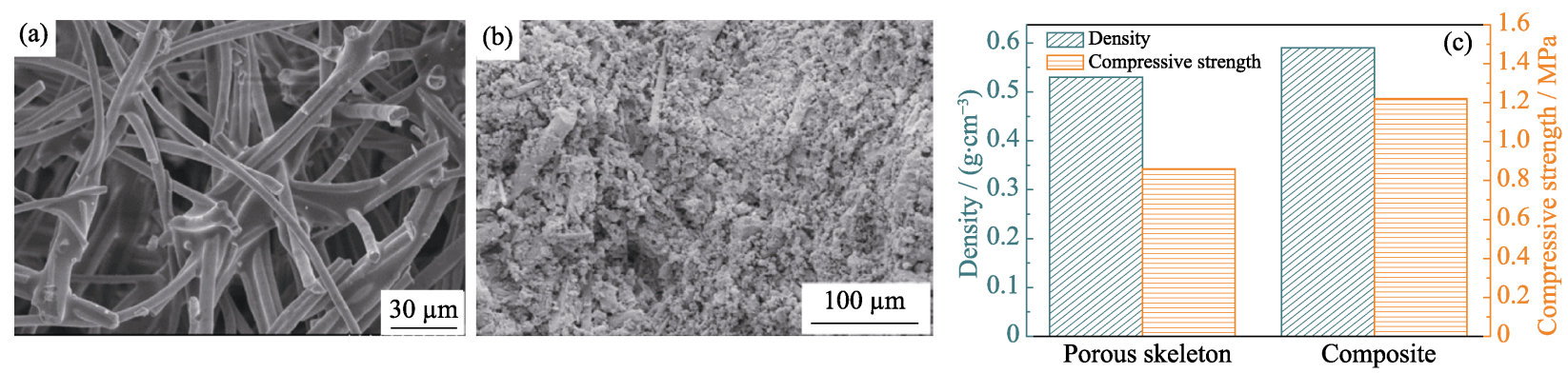

图 7 氧化锆纤维多孔骨架及其增强的氧化铝-氧化硅气凝胶复合材料 ${ }^{[37]}$

Fig. 7 Zirconia fiber-based porous skeleton and the alumina-silica aerogel composite reinforced by the skeleton ${ }^{[37]}$ Micro morphology of (a) skeleton and (b) composite, and (c) their mechanical properties
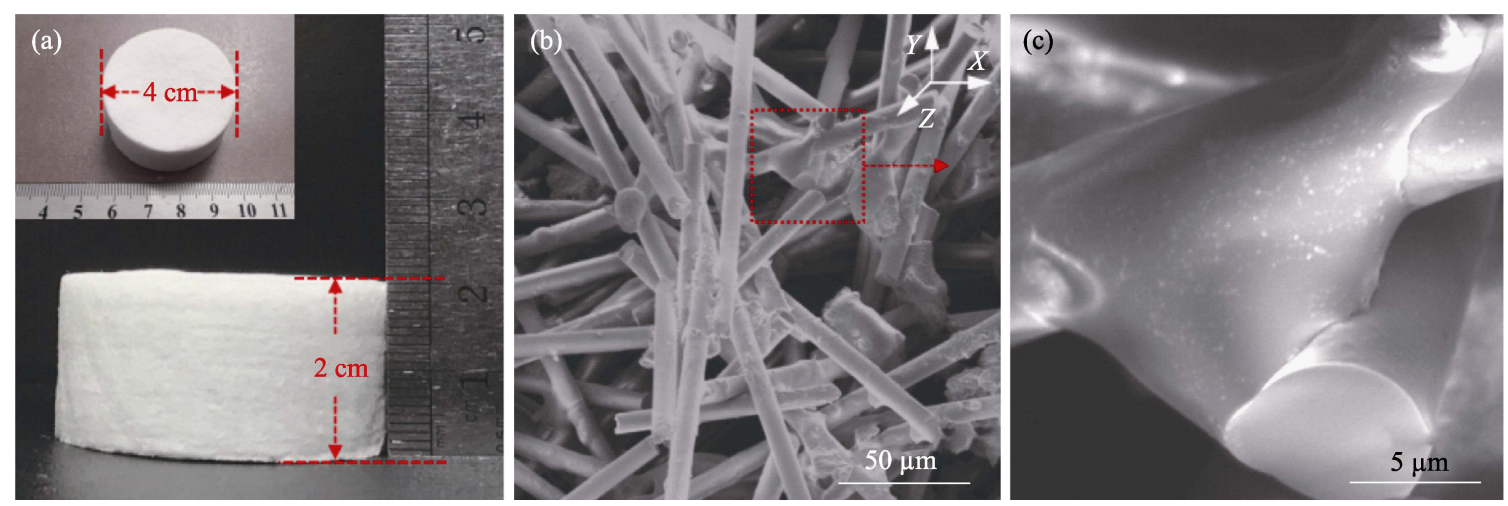

图 8 石英纤维多孔骨架宏观形貌 $(a)$ 和微观形貌 $(b, c)^{[33]}$

Fig. 8 The macro (a) and micro morphologies $(b, c)$ of the quartz fiber-based porous skeleton ${ }^{[33]}$ 
纤维䏨(杨氏模量为 $0.04 \mathrm{MPa}$ )强度显著提高。在 压缩载荷下，复合材料经历了线性(0 8\%应变)、 屈服((8 30)\%应变)和密实化(应变大于 $30 \%)$ 三个 变形阶段。

多孔骨架本身具有一定的强度和较低的热导率, 因此其对于氧化铝气凝胶是一种合适的增强体，能 够制备出力学性能(如压缩强度)较好的复合材料。 但是，在多孔骨架的制备过程中，通常需要使用粘 接剂将纤维结合在一起, 一定程度上增加了纤维骨 架之间的固体热传导。如何用更为简单的工艺制备 多孔骨架并减少骨架连接处的热桥效应, 以降低多 孔骨架本身的热导率, 是未来的重要研究内容。

\section{3 纤维增强}

采用纤维与氧化铝溶胶直接复合是制备氧化铝 气凝胶复合材料的常用方法。根据长径比, 纤维可 分为短纤维和长纤维两种。其中, 短纤维一般以类 似于晶须的引入方式, 通过机械搅拌分散在氧化铝 溶胶中; 长纤维内部含有大量孔隙, 氧化铝溶胶可 通过浸渍过程渗入其中。

$\mathrm{Yu}$ 等 ${ }^{[55]}$ 将氧化铝-氧化硅短切纤维在高速搅拌 下分散于溶胶中, 制备了氧化铝-氧化硅气凝胶复 合材料, 其强度随纤维质量分数增大而提高(图 9(a))。 但是, 当纤维含量过高时, 不利于纤维和气凝胶之 间的结合，复合材料的强度反而下降。
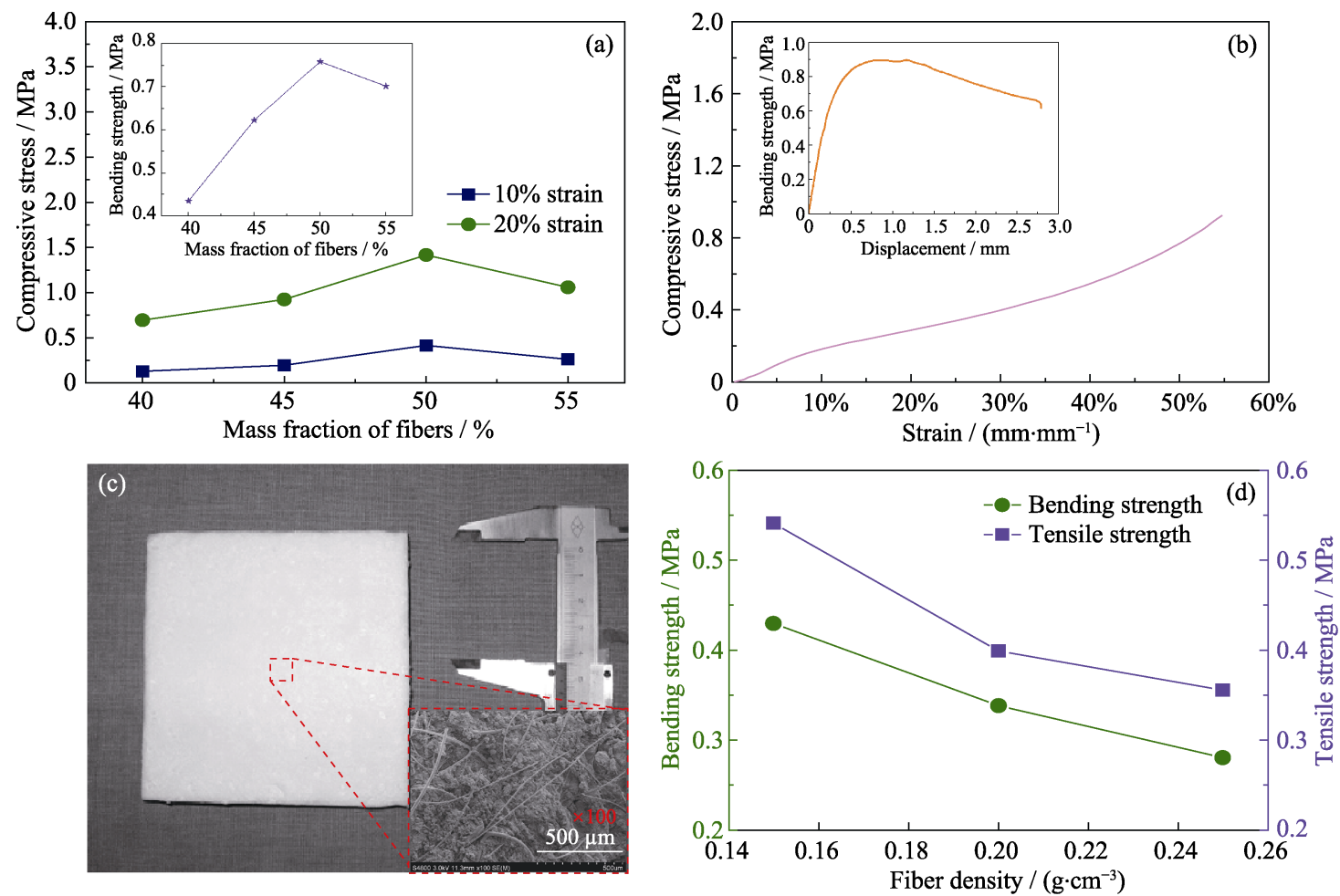

图 9 纤维增强氧化铝(氧化铝-氧化硅)气凝胶复合材料 ${ }^{[55-57]}$

Fig. 9 Fiber reinforced alumina (alumina-silica) aerogel composites ${ }^{[55-57]}$

(a) Influence of mass fraction of fibers on mechanical properties ${ }^{[55]}$; (b) Behavior of composites under compressive and bending loadings ${ }^{[56]}$; (c) Morphology and (d) bending/tensile strengths of composites with different fiber densities ${ }^{[57]}$ 


\section{3 氧化铝气凝胶复合材料的隔热性能}

纯氧化铝气凝胶具有很低的密度和纳米孔结构, 显著抑制了固态和气态热传导, 因而常温热导率较 低, 但在高温下的红外辐射透过率很高 ${ }^{[59]}$, 必须引 入能够抑制红外辐射的物质(如纤维和遮光剂)以降 低其高温热导率。

\section{1 纤维对复合材料隔热性能的影响}

研究表明, 纤维不仅能够提高气凝胶的力学性 能, 而且能够对红外辐射进行散射和吸收 ${ }^{[59-62]}$, 从 而提高氧化铝气凝胶的高温隔热性能。

高庆福等 ${ }^{[56,63]}$ 制备了 $1000{ }^{\circ} \mathrm{C}$ 热导率(水流量平 板法)为 $0.0685 \mathrm{~W} /(\mathrm{m} \cdot \mathrm{K})$ 的陶瓷纤维增强氧化铝气 凝胶复合材料。在石英灯单面加热 (热面温度 $1000{ }^{\circ} \mathrm{C}$, 加热 $\left.10 \mathrm{~min}\right)$ 试验中, 复合材料的冷面温度 仅为 $484{ }^{\circ} \mathrm{C}$ (图 10), 表现出良好的隔热性能。

武纬 ${ }^{[57]}$ 发现, 随着纤维体积密度增大, 莫来石 纤维增强氧化铝-氧化硅气凝胶复合材料在高温段 的热导率显著降低, 其在 $800 、 1000{ }^{\circ} \mathrm{C}$ 时的热导率 (水流量平板法)分别仅为 $0.047 、 0.062 \mathrm{~W} /(\mathrm{m} \cdot \mathrm{K})$ (图 11); 在石英灯单面加热(热面温度 $1000{ }^{\circ} \mathrm{C}$, 加 热 $11 \mathrm{~min}$ )测试过程中, 复合材料的冷面温度较低。
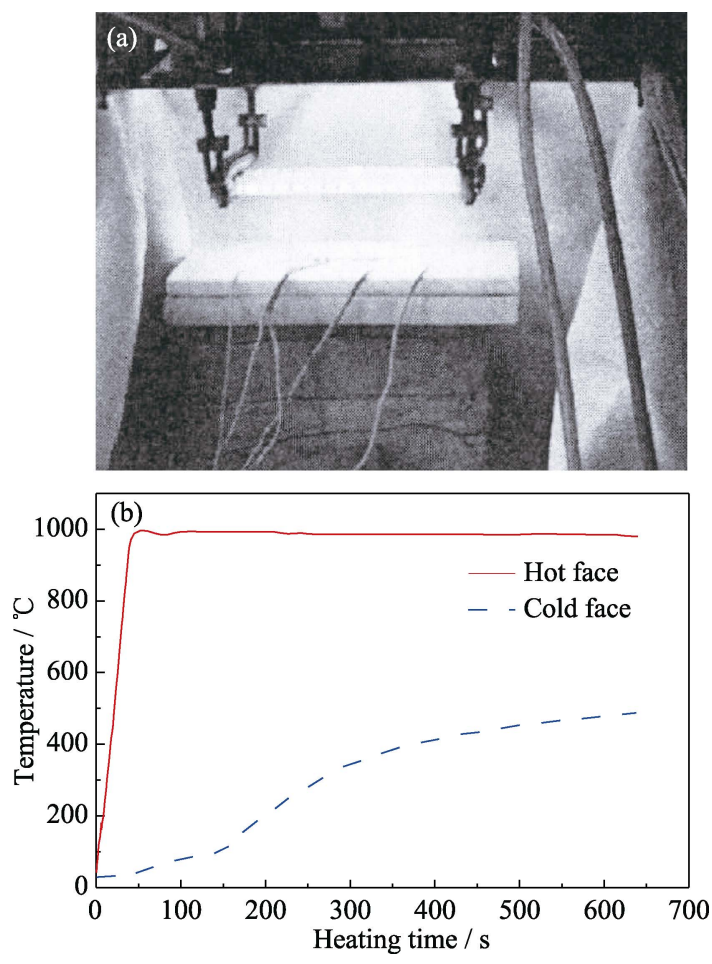

图 10 陶瓷纤维增强氧化铝气凝胶复合材料(厚度 $15 \mathrm{~mm}$ ) 的石英灯单面加热试验现场(a)和温度曲线 $(b)^{[56]}$

Fig. 10 Test site (a) and temperature curves (b) of the ceramic fiber reinforced alumina aerogel composite $(15 \mathrm{~mm}$ in thickness) heated by a quartz lamp apparatus ${ }^{[56]}$

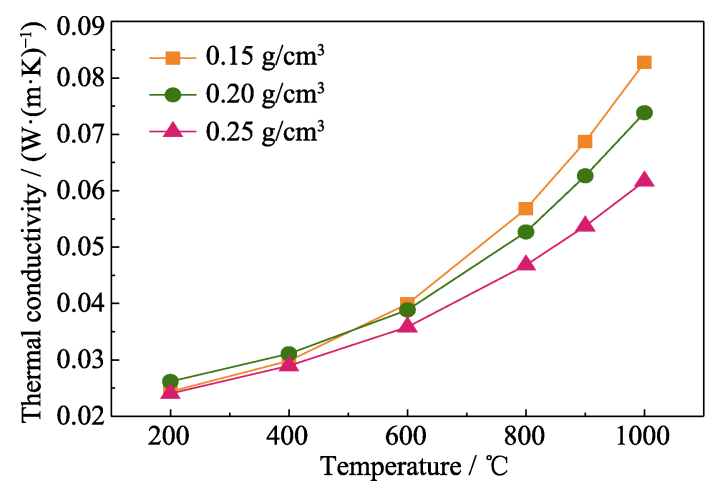

图 11 莫来石纤维增强氧化铝一氧化硅气凝胶复合材料的高 温热导率 ${ }^{[57]}$

Fig. 11 High-temperature thermal conductivities of mullite fiber reinforced alumina-silica aerogel composites ${ }^{[57]}$

Yang 等 ${ }^{[64]}$ 发现采用高压浸渍方式能够减少气凝胶 中的大孔数量, 从而进一步降低氧化铝-氧化硅气 凝胶复合材料的热导率 $\left(1000 、 1100{ }^{\circ} \mathrm{C}\right.$ 时分别为 $0.061 、 0.071 \mathrm{~W} /(\mathrm{m} \cdot \mathrm{K})$, 水流量平板法 $)$ 。

Wang 等 ${ }^{[65]}$ 以石英纤维织物作为增强相制备了 氧化铝一氧化硅气凝胶复合材料, 并采用厚度为 $25 \mathrm{~mm}$ 的复合材料进行了石英灯单面加热(热面温 度 $1200{ }^{\circ} \mathrm{C}$, 加热 $25 \mathrm{~min}$ )试验, 试验结束时的冷面 温度为 $733{ }^{\circ} \mathrm{C}$ 。

碳纤维增强的氧化铝气凝胶复合材料在高温下 同样具有较低的热导率。Zhong 等 ${ }^{[66]}$ 以六水氯化铝、 间苯二酚、甲醛和碳纤维毡为主要原料, 经高温裂 解得到碳纤维增强碳/氧化铝气凝胶复合材料, 1200、1400、 $1600{ }^{\circ} \mathrm{C}$ 热导率(氩气气氛，激光闪射 法)分别为 $0.18 、 0.23 、 0.284 \mathrm{~W} /(\mathrm{m} \cdot \mathrm{K})$ 。 $\mathrm{Li}$ 等 ${ }^{[67]}$ 以 氯化铝为先驱体, 制备了碳纤维增强氧化铝气凝胶 复合材料, 复合材料的常温、 $1000{ }^{\circ} \mathrm{C}$ 热导率(氩气气 氛，激光闪射法)分别为 $0.081 、 0.330 \mathrm{~W} /(\mathrm{m} \cdot \mathrm{K})$ 。

\section{2 遮光剂对复合材料隔热性能的影响}

由于纤维对红外辐射的抑制作用有限, 研究人 员在氧化铝气凝胶复合材料中引入耐高温的遮光剂 组分(如氧化钛、氧化锆、碳化硅等), 以提高其对红 外辐射波的散射和吸收能力, 进一步降低高温热 导率。

周洁洁等 ${ }^{[68]}$ 将红外遮光剂加入到溶胶中, 制备 了常温热导率(热流计法)为 $0.03080 \mathrm{~W} /(\mathrm{m} \cdot \mathrm{K})$ 的氧化 铝-氧化硅气凝胶复合材料(图 12(a))。在石英灯单面 加热(热面温度 $1200{ }^{\circ} \mathrm{C}$, 加热 $30 \mathrm{~min}$ )试验中, 含遮 光剂的复合材料的冷面温度显著低于莫来石纤维毡 和不含遮光剂的复合材料(图 12(b))。孙晶晶等 ${ }^{[53]}$ 将碳化硅分散在氧化铝溶胶中, 再浸渍隔热瓦制备 得到隔热瓦复合氧化铝气凝胶复合材料。采用石英 
灯对材料进行 $1400{ }^{\circ} \mathrm{C}$ 单面加热 $30 \mathrm{~min}$, 加入和未 加入碳化硅的复合材料的冷面温度分别为 830 、

$870{ }^{\circ} \mathrm{C}$, 表明引入遮光剂提高了高温隔热效果。

近年来有研究报导了引入氧化钛颗粒对氧化铝 气凝胶复合材料结构和性能的影响规律。 $\mathrm{Zu}$ 等 ${ }^{[47]}$ 将氧化钛颗粒(直径为 $250 \mathrm{~nm}$ 左右)分散到氧化铝溶 胶中，再浸渍莫来石纤维段得到氧化铝气凝胶复合 材料, 其高温热导率(水流量平板法)显著低于莫来
石纤维毡以及不含氧化钛的复合材料(图 13(a))。Zou 等 ${ }^{[54]}$ 通过冷冻干燥和烧结工艺将亚微米尺寸的氧 化钛颗粒分散于莫来石纤维多孔骨架中，再浸渍氧 化铝-氧化硅溶胶，制备得到氧化铝-氧化硅气凝胶 复合材料。适量氧化钛的引入使复合材料在 $800{ }^{\circ} \mathrm{C}$ 以上的热导率下降(图 13(b))。在丁烷火焰加热下, 复 合材料的冷面温度显著低于莫来石纤维毡(图 13(c))。 亚微米尺寸的氧化钛颗粒对 $800 \sim 1200{ }^{\circ} \mathrm{C}$ 下的红外
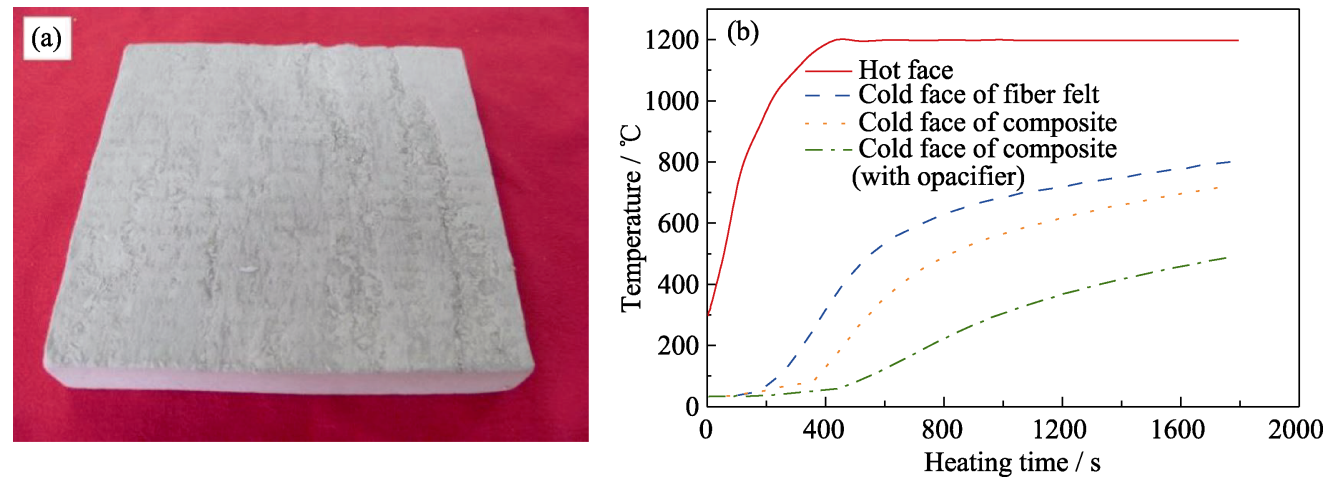

图 12 加入遮光剂的莫来石纤维廷增强氧化铝-氧化硅气凝胶复合材料 ${ }^{[68]}$

Fig. 12 Opacifier embedded mullite fiber felt reinforced alumina-silica aerogel composite ${ }^{[68]}$

(a) Macro morphology; (b) Temperature curves of the composite (20 $\mathrm{mm}$ in thickness) during one-face heating test
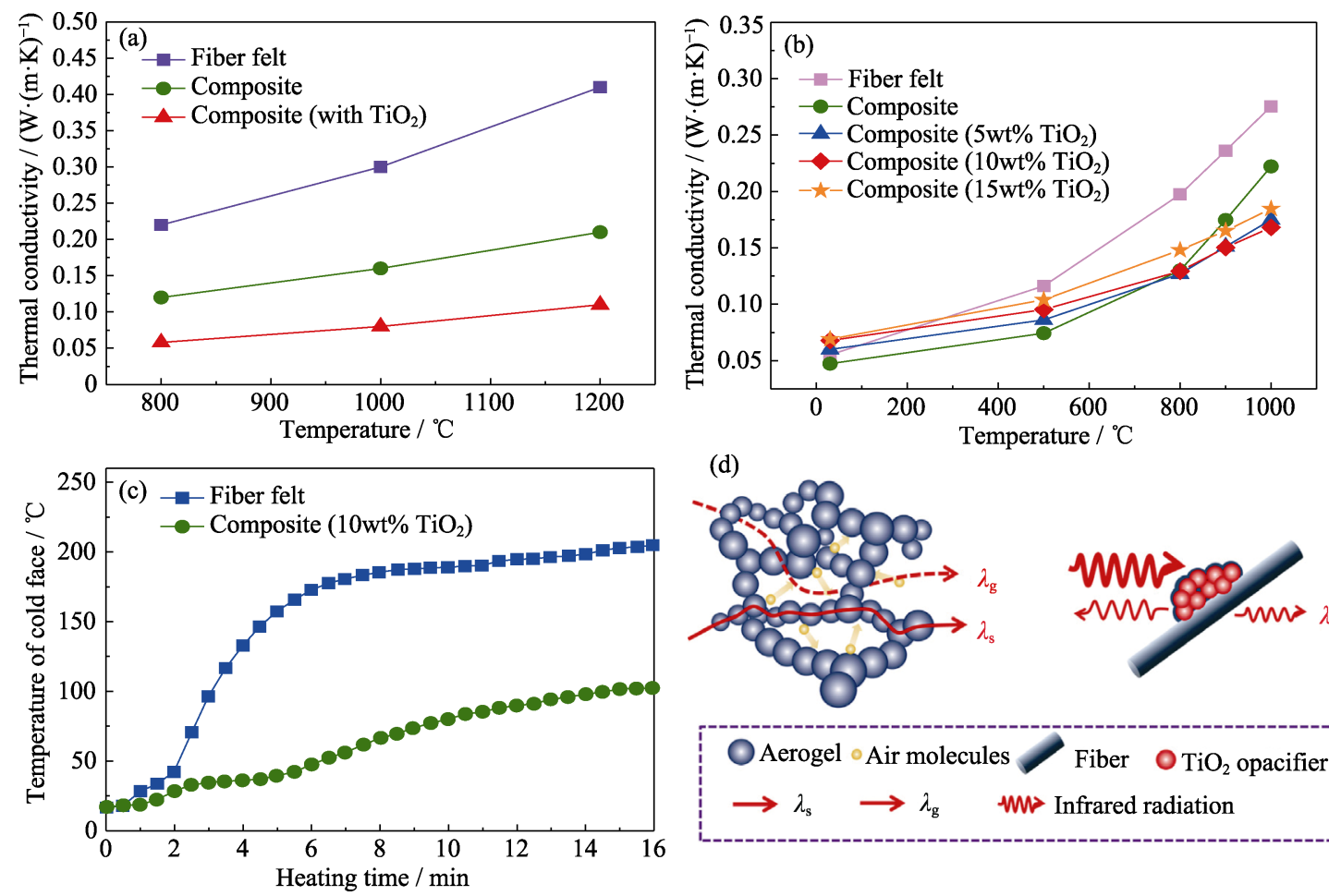

(d)

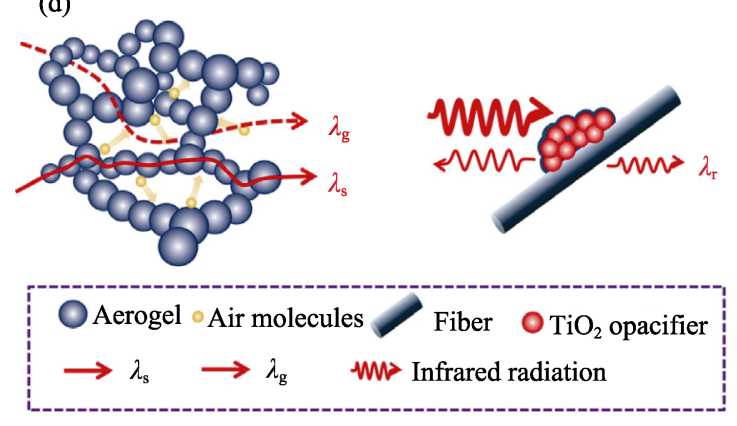

图 13 引入氧化钛的莫来石纤维增强氧化铝气凝胶复合材料的高温热导率 $(\mathrm{a})^{[47]}$, 不同氧化钛含量复合材料的 高温热导率(b) ${ }^{[54]}$, 含氧化钛的复合材料(厚度为 $20 \mathrm{~mm}$ )在丁烷火焰加热 $\left(1300{ }^{\circ} \mathrm{C}, 15 \mathrm{~min}\right)$ 下的冷面温度(c) $)^{[54]}$, 复合材料的传热机制 $\left(\lambda_{\mathrm{s}} 、 \lambda_{\mathrm{g}}\right.$ 分别为固态、气态热导率 $)(\mathrm{d})^{[54]}$

Fig. 13 The mullite fiber felt reinforced alumina-silica aerogel composite doped with $\mathrm{TiO}_{2}$

(a) High-temperature thermal conductivity ${ }^{[47]}$; (b) High-temperature thermal conductivity of composites with different content of $\mathrm{TiO}_{2}{ }^{[54]}$;

(c) Temperature (cold-face) of the composite (doped with $10 \mathrm{wt} \% \mathrm{TiO}_{2}$ ) fired by the flame of butane spray gun at $1300{ }^{\circ} \mathrm{C}$ for $15 \mathrm{~min}^{[54]}$; (d) $\mathrm{Heat}$ transfer mechanism of the composite $\left(\lambda_{\mathrm{s}} \text { and } \lambda_{\mathrm{g}} \text { refering to solid and gaseous thermal conductivity, respectively }\right)^{[54]}$ 

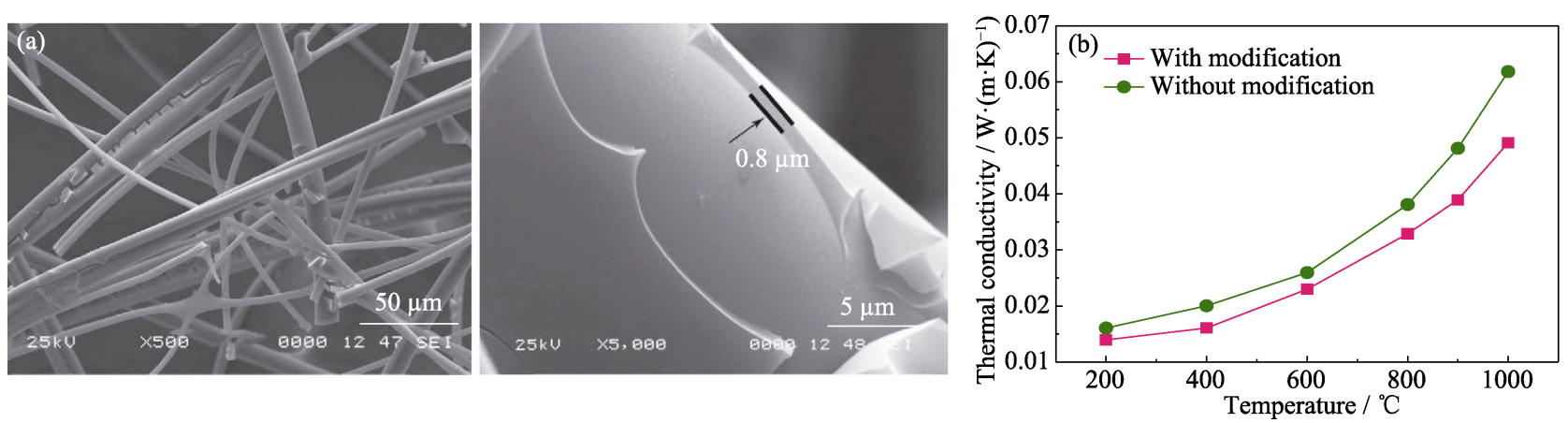

图 14 碳化硅改性莫来石纤维的微观形貌(a)和以改性莫来石纤维作为增强相的 氧化铝-氧化硅气凝胶复合材料的热导率(b) ${ }^{[69]}$

Fig. 14 (a) Micro morphology of SiC modified mullite fibers; (b) Thermal conductivity of the alumina-silica aerogel composite reinforced by $\mathrm{SiC}$ modified mullite fibers ${ }^{[69]}$

辐射波具有显著的散射和吸收效应，从而大幅抑制 了辐射传热(图 13(d))。

$\mathrm{Xu}$ 等 ${ }^{[69]}$ 以聚碳硅烷溶液浸渍莫来石纤维, 经高 温裂解工艺在纤维表面制备了碳化硅涂层(图 14(a)), 再以纤维为增强相, 制备了氧化铝-氧化硅气凝胶 复合材料。碳化硅涂层显著提高了纤维对 2.5 7.5 $\mu \mathrm{m}$ 波长范围内红外辐射波的比消光系数, 使复合材料 的热导率(水流量平板法)显著降低(图 14(b)), $1000{ }^{\circ} \mathrm{C}$ 时仅为 $0.049 \mathrm{~W} /(\mathrm{m} \cdot \mathrm{K})$ 。

除了机械分散或纤维改性的方法, 采用原位掺 杂方式能够在氧化铝气凝胶中引入纳米尺度的遮光 剂 ${ }^{[58,70]}$ 。朱召贤等 ${ }^{[88]}$ 在氧化铝一氧化硅溶胶中加入 四氯化钛、氯氧化锆, 再浸渍莫来石纤维, 经超临界 干燥分别制备了氧化钛、氧化锆掺杂的氧化铝-氧化 硅气凝胶复合材料。两种复合材料在 $1050{ }^{\circ} \mathrm{C}$ 时的热 导率(测试方法未注明)分别为 $0.084 、 0.076 \mathrm{~W} /(\mathrm{m} \cdot \mathrm{K})$, 相对于未掺杂遮光剂的复合材料明显下降。Gao 等 ${ }^{[70]}$ 亦发现氧化铝-氧化钛气凝胶复合材料具有较低的 高温热导率 $\left(1000{ }^{\circ} \mathrm{C}\right.$ 时为 $0.136 \mathrm{~W} /(\mathrm{m} \cdot \mathrm{K})$, 水流量平 板法)。

\section{3 复合材料的典型热导率}

由图 15 可知, 纯氧化铝气凝胶的热导率随温度 升高而急剧上升, 其 $1000{ }^{\circ} \mathrm{C}$ 热导率可达常温热导 率的 10 倍以上。引入纤维等增强相和遮光剂之后制 备的氧化铝气凝胶复合材料的常温热导率 $(0.022$ $0.068 \mathrm{~W} /(\mathrm{m} \cdot \mathrm{K}))$ 有所上升, 主要原因是这些组分一 定程度上增加了固态传热。相比于纯氧化铝气凝胶, 复合材料的高温热导率显著下降, 综合隔热性能大 幅提高。综上所述, 目前制备的氧化铝气凝胶复合 材料的常温热导率仍然偏高, 其高温热导率随温度 升高的趋势仍然较为明显， $1200{ }^{\circ} \mathrm{C}$ 热导率上升至 $0.09 \mathrm{~W} /(\mathrm{m} \cdot \mathrm{K})$ 以上。但是, 通过引入遮光剂, 氧化铝

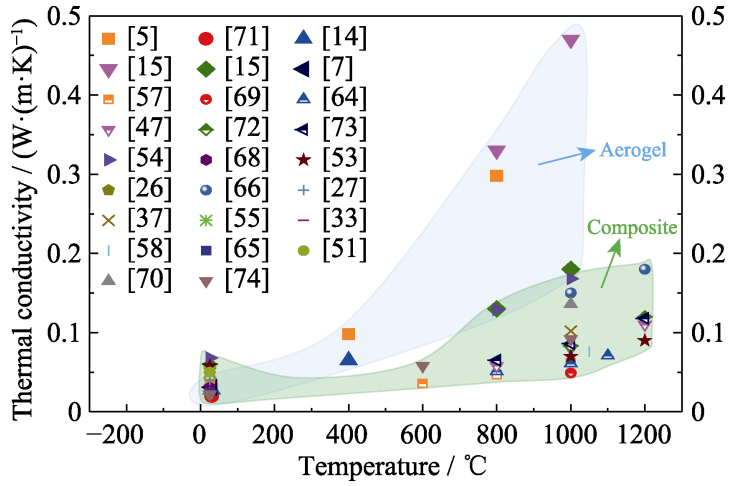

图 15 典型的纯氧化铝气凝胶及其隔热复合材料的热导 率值

Fig. 15 Thermal conductivity of typical pristine alumina aerogels and alumina aerogel insulation composites

气凝胶复合材料在 $1000{ }^{\circ} \mathrm{C}$ 时的热导率可降低至 $0.049 \mathrm{~W} /(\mathrm{m} \cdot \mathrm{K})^{[69]}$, 表明复合材料的热导率还有较 大的优化空间。后续的工作中, 需要深入研究复合 材料各组分间的协同传热机制，调整氧化铝气凝胶 的孔结构和体积分数、遮光剂的种类和体积分数等 关键参数, 以进一步降低氧化铝气凝胶复合材料在 整个温度范围内的热导率。

\section{4 总结与展望}

本文对耐高温氧化铝气凝胶的制备、氧化铝气 凝胶隔热复合材料的制备及性能等方面的最新研究 进展进行了总结。通过元素掺杂、沉积改性等方法, 氧化铝气凝胶的热稳定性得到了明显改善; 在氧化 铝气凝胶中引入纤维等增强相能够大幅提高其力学 性能; 纤维和遮光剂的协同作用, 提高了氧化铝气 凝胶抑制红外辐射的能力, 显著降低了高温热导 率。针对目前存在的问题, 后续可能的研究方向包括:

1)氧化铝气凝胶的耐温性提升。目前制备的氧 
化铝气凝胶在高温 $\left(1200{ }^{\circ} \mathrm{C}\right.$ 以上 $)$ 下仍然会发生显 著的体积收缩和孔结构破坏。研究表明, 提高氧化 铝气凝胶的骨架强度能够降低其高温下的收缩 ${ }^{[7]}$; 气凝胶颗粒形貌对热稳定性有显著影响 ${ }^{[18]}$ 。因此, 尽量采用简单工艺, 对氧化铝气凝胶的骨架、微观 形貌进行精细调控, 再引入适当异质元素(如硅), 是提高其热稳定性的有效措施，有待进一步研究。

2)氧化铝气凝胶复合材料的隔热性能优化。目 前, 氧化铝气凝胶复合材料在整个温度范围的热导 率仍然相对偏高。首先需要进一步调控氧化铝气凝 胶的密度、颗粒尺寸/形貌, 使其具有合适的密度和 小的孔径, 以进一步降低复合材料的气态热导率。 另一方面, 需要采用更为简单、高效的方法, 将合适 种类、尺寸和含量的遮光剂均匀引入至氧化铝气凝 胶复合材料中，以进一步降低高温热导率。

3)复合材料在高温下结构和性能的演化。高温 环境中, 复合材料中的氧化铝气凝胶和增强相之间 会发生相互作用，将对复合材料的结构和性能产生 显著影响。主要的研究方式有两种：将复合材料热 处理后进行尺寸、微观形貌、晶型、性能等的表征 ${ }^{[75]}$; 在加热条件下实时观察复合材料尺寸、微观结构和 性能的变化。

随着技术的不断发展和完善，氧化铝气凝胶复 合材料将在高温隔热领域发挥其优势并逐步实现 应用。

\section{参考文献:}

[1] UYANNA O, NAJAFI H. Thermal protection systems for space vehicles: a review on technology development, current challenges and future prospects. Acta Astronautica, 2020, 176: 341-356.

[2] KOEBEL M M, RIGACCI A, ACHARD P. Aerogels Handbook, 2010: 607-632.

[3] CAI H, JIANG Y, FENG J, et al. Preparation of silica aerogels with high temperature resistance and low thermal conductivity by monodispersed silica sol. Materials \& Design, 2020, 191: 108640.

[4] WANG F, DOU L, DAI J, et al. In situ synthesis of biomimetic silica nanofibrous aerogels with temperature-invariant superelasticity over one million compressions. Angewandte Chemie International Edition, 2020, 59(21): 8285-8292.

[5] POCO J F, JR J H S, HRUBESH L W. Synthesis of high porosity, monolithic alumina aerogels. Journal of Non-Crystalline Solids, 2001, 285: 57-63.

[6] 高庆福, 张长瑞, 冯坚, 等. 低密度、块状氧化铝气凝胶制备. 无机化学学报, 2008, 24(9): 1456-1460.

[7] ZU G, SHEN J, ZOU L, et al. Nanoengineering super heat-resistant, strong alumina aeroegels. Chemistry of Materials, 2013, 25: 4757-4764.

[8] ZU G, SHEN J, ZOU L, et al. Highly thermally stable zirconia/ silica composite aerogels prepared by supercritical deposition. Microporous and Mesoporous Materials, 2017, 238: 90-96.

[9] SU L, WANG H, NIU M, et al. Ultralight, recoverable, and high temperature-resistant $\mathrm{SiC}$ nanowire aerogel. ACS Nano, 2018, 12(4): 3103-3111.

[10] XU X, ZHANG Q, HAO M, et al. Double-negative-index ceramic aerogels for thermal superinsulation. Science, 2019, 363: 723-727.

[11] 艾素芬, 孙言，雷尧飞，等. 低密度气凝胶的高温结构变化及其 耐温性研究. 北京化工大学学报, 2019, 46(1): 63-68.

[12] CAI H, JIANG Y, FENG J, et al. Nanostructure evolution of silica aerogels under rapid heating from $600{ }^{\circ} \mathrm{C}$ to $1300{ }^{\circ} \mathrm{C}$ via in-situ TEM observation. Ceramics International, 2020, 46(8): 12489-12498.

[13] BAUMANN T F, GASH A E, CHINN S C, et al. Synthesis of high-surface-area alumina aerogels without the use of alkoxide precursors. Chemistry of Materials, 2005, 17: 395-401.

[14] ZU G, SHEN J, WEI X, et al. Preparation and characterization of monolithic alumina aerogels. Journal of Non-Crystalline Solids, 2011, 357: 2903-2906

[15] WANG W, ZHANG Z, ZU G, et al. Trimethylethoxysilanemodified super heat-resistant alumina aerogels for high-temperature thermal insulation and adsorption applications. RSC Advances, 2014, 4: 54864-54871

[16] 温培刚, 巢雄宇, 袁武华, 等. 耐高温氧化铝气凝胶研究进展. 材料导报, 2016, 30(8): 51-56.

[17] 李华金鍂, 赵春林, 陈俊勇, 等. 氧化铝气凝胶研究进展. 金属世 界, 2018(4): 27-33.

[18] PAKHARUKOVA V P, SHALYGIN A S, GERASIMOV E Y, et al. Structure and morphology evolution of silica-modified pseudoboehmite aerogels during heat treatment. Journal of Solid State Chemistry, 2016, 233: 294-302.

[19] BARARPOUR S T, KARAMI D, MAHINPEY N. Investigation of the effect of alumina-aerogel support on the $\mathrm{CO}_{2}$ capture performance of $\mathrm{K}_{2} \mathrm{CO}_{3}$. Fuel, 2019, 242: 124-132.

[20] HURWITZ F I, GALLAGHER M, OLIN T C, et al. Optimization of alumina and aluminosilicate aerogel structure for high-temperature performance. International Journal of Applied Glass Science, 2014 5(3): 276-286.

[21] YOROV K E, YAPRYNTSEV A D, BARANCHIKOV A E, et al. Luminescent alumina-based aerogels modified with tris(8-hydroxyquinolinato)aluminum. Journal of Sol-Gel Science and Technology, 2018, 86: 400-409.

[22] WEN S, REN H, ZHU J, et al. Fabrication of $\mathrm{Al}_{2} \mathrm{O}_{3}$ aerogel- $\mathrm{SiO}_{2}$ fiber composite with enhanced thermal insulation and high heat resistance. Journal of Porous Materials, 2018, 26: 1027-1034.

[23] ARAVIND P R, MUKUNDAN P, PILLAI P K, et al. Mesoporous silica-alumina aerogels with high thermal pore stability through hybrid Sol-Gel route followed by subcritical drying. Microporous and Mesoporous Materials, 2006, 96(1): 14-20.

[24] HAYASE G, NONOMURA K, HASEGAWA G, et al. Ultralowdensity, transparent, superamphiphobic boehmite nanofiber aerogels and their alumina derivatives. Chemistry of Materials, 2015, 27(1): $3-5$.

[25] 冯坚, 高庆福, 武纬, 等. 硅含量对 $\mathrm{Al}_{2} \mathrm{O}_{3}-\mathrm{SiO}_{2}$ 气凝胶结构和性 能的影响. 无机化学学报, 2009, 25(10): 1758-1763.

[26] WU X, SHAO G, SHEN X, et al. Novel $\mathrm{Al}_{2} \mathrm{O}_{3}-\mathrm{SiO}_{2}$ composite aerogels with high specific surface area at elevated temperatures with different alumina/silica molar ratios prepared by a nonalkoxide Sol-Gel method. RSC Advances, 2016, 6: 5611-5620.

[27] HOU X, ZHANG R, FANG D. Novel whisker-reinforced $\mathrm{Al}_{2} \mathrm{O}_{3}$ $\mathrm{SiO}_{2}$ aerogel composites with ultra-low thermal conductivity. Ceramics International, 2017, 43: 9547-9551.

[28] YANG J, WANG Q, WANG T, et al. Facile one-step precursorto-aerogel synthesis of silica-doped alumina aerogels with high specific surface area at elevated temperatures. Journal of Porous Materials, 2017, 24: 889-897.

[29] KARAMI D, MAHINPEY N. Utilization of alumina aerogel as high surface area support for the fabrication of oxygen carriers in the chemical looping combustion process. Energy \& Fuels, 2019, 33: 5408-5414.

[30] MIZUSHIMA Y, HORI M. Preparation of heat-resistant alumina aerogels. Journal of Materials Research, 1993, 8(11): 2993-2999.

[31] OSAKI T, NAGASHIMA K, WATARI K, et al. Silica-doped alumina cryogels with high thermal stability. Journal of NonCrystalline Solids, 2007, 353: 2436-2442. 
[32] JI X, ZHOU Q, QIU G, et al. Synthesis of an alumina enriched $\mathrm{Al}_{2} \mathrm{O}_{3}-\mathrm{SiO}_{2}$ aerogel: reinforcement and ambient pressure drying. Journal of Non-Crystalline Solids, 2017, 471: 160-168.

[33] YU H, JIANG Y, LU Y, et al. Quartz fiber reinforced $\mathrm{Al}_{2} \mathrm{O}_{3}-\mathrm{SiO}_{2}$ aerogel composite with highly thermal stability by ambient pressure drying. Journal of Non-Crystalline Solids, 2019, 505: 79-86.

[34] HORIUCHI T, OSAKI T, SUGIYAMA T, et al. Maintenance of large surface area of alumina heated at elevated temperatures above $1300{ }^{\circ} \mathrm{C}$ by preparing silica-containing pseudoboehmite aerogel. Journal of Non-Crystalline Solids, 2001, 291: 187-198.

[35] HURWITZ F I, GUO H, ROGERS R B, et al. Influence of Ti addition on boehmite-derived aluminum silicate aerogels: structure and properties. Journal of Sol-Gel Science and Technology, 2012, 64: $367-374$

[36] WU X, SHAO G, CUI S, et al. Synthesis of a novel $\mathrm{Al}_{2} \mathrm{O}_{3}-\mathrm{SiO}_{2}$ composite aerogel with high specific surface area at elevated temperatures using inexpensive inorganic salt of aluminum. Ceramics International, 2016, 42: 874-882.

[37] ZHANG R, YE C, WANG B. Novel $\mathrm{Al}_{2} \mathrm{O}_{3}-\mathrm{SiO}_{2}$ aerogel/porous zirconia composite with ultralow thermal conductivity. Journal of Porous Materials, 2018, 25: 171-178.

[38] 张恩爽, 黄红岩, 刘蹈, 等. 结构强健的 $\mathrm{Al}_{2} \mathrm{O}_{3}-\mathrm{SiO}_{2}$ 气凝胶的 制备及可重复使用性能. 高等学校化学学报, 2019, 40(12): 2566-2573.

[39] PENG F, JIANG Y, FENG J, et al. A facile method to fabricate monolithic alumina-silica aerogels with high surface areas and good mechanical properties. Journal of the European Ceramic Society, 2020, 40(6): 2480-2488.

[40] TOKUBOME Y, NAKANISHI K, HANADA T. Effect of La addition on thermal microstructural evolution of macroporous alumina monolith prepared from ionic precursors. Journal of the Ceramic Society of Japan, 2009, 117(1363): 351-355.

[41] YANG J, WANG Q, WANG T, et al. Rapid preparation process, structure and thermal stability of lanthanum doped alumina aerogels with a high specific surface area. RSC Advances, 2016, 6: 26271-26279.

[42] SUN X, WU Y, WANG Y, et al. Investigation of the effect of lanthanum oxide on the thermal stability of alumina aerogel. Journal of Porous Materials, 2018, 26: 327-333.

[43] AL-YASSIR N, MAO R L V. Thermal stability of alumina aerogel doped with yttrium oxide, use as a catalyst support for the thermocatalytic cracking (TCC) process: an investigation of its textural and structural properties. Applied Catalysis, 2007, 317: 275-283.

[44] 周洁洁, 陈晓红, 宋怀河, 等. 氧化钎掺杂对 $\mathrm{Al}_{2} \mathrm{O}_{3}$ 块状气凝胶 结构与性能的影响. 硅酸盐通报, 2010, 29(5): 1002-1006.

[45] 孙雪峰, 吴玉胜, 李来时, 等. 锶掺杂对氧化铝气凝胶高温热稳 定性的影响. 功能材料, 2018, 8(9): 09078-09081, 09086.

[46] SHI Z, GAO H, WANG X, et al. One-step synthesis of monolithic micro-nano yttria stabilized $\mathrm{ZrO}_{2}-\mathrm{Al}_{2} \mathrm{O}_{3}$ composite aerogel. Microporous and Mesoporous Materials, 2018, 259: 26-32.

[47] ZU G, SHEN J, WANG W, et al. Robust, highly thermally stable, core-shell nanostructured metal oxide aerogels as high-temperature thermal superinsulators, adsorbents, and catalyst. Chemistry of Materials, 2014, 26: 5761-5772.

[48] MIZUSHIMA Y, HORI M. Preparation and properties of alumina-organic compound aerogels. Journal of Non-Crystalline Solids, 1994, 170: 215-222.

[49] YAKOVLEVA I V, VOLODINA A M, ZAIKOVSKIIA V I, et al. Stabilizing effect of the carbon shell on phase transformation of the nanocrystalline alumina particles. Ceramics International, 2018, 44: 4801-4806.

[50] MIZUSHIMA Y, HORI M. Preparation of an alumina aerogel with $\mathrm{SiC}$ whisker inclusion. Journal of the European Ceramic Society, 1994, 14: 117-121.

[51] 隋超. 纤维素掺杂 $\mathrm{SiO}_{2}$ 与 $\mathrm{Al}_{2} \mathrm{O}_{3}$ 柔性气凝胶的制备及性能表 征. 哈尔滨: 哈尔滨工业大学博士学位论文, 2015 .

[52] 曹凤朝. 高强度氧化铝气凝胶复合材料的制备研究. 南京: 东 南大学硕士学位论文, 2015 .
[53] 孙晶晶, 胡子君, 吴文军, 等. 氧化铝气凝胶复合高温隔热瓦的 制备及性能. 宇航材料工艺, 2017(3): 33-36, 41.

[54] ZOU W, WANG X, WU Y, et al. Opacifier embedded and fiber reinforced alumina-based aerogel composites for ultra-high temperature thermal insulation. Ceramics International, 2019, 45(1): $644-650$.

[55] YU Y, PENG K, FANG J, et al. Mechanical and thermal conductive properties of fiber-reinforced silica-alumina aerogels. Applied Ceramic Technology, 2018, 15(5): 1138-1145.

[56] 高庆福. 纳米多孔 $\mathrm{SiO}_{2} 、 \mathrm{Al}_{2} \mathrm{O}_{3}$ 气凝胶及其高效隔热复合材料的 研究. 长沙: 国防科技大学博士学位论文, 2009.

[57] 武纬. $\mathrm{Al}_{2} \mathrm{O}_{3}-\mathrm{SiO}_{2}$ 气凝胶及其隔热复合材料的制备与性能研究. 长沙: 国防科技大学硕士学位论文, 2008 .

[58] ZHU Z, WANG F, YAO J, et al. High-temperature insulation property of opacifier-doped $\mathrm{Al}_{2} \mathrm{O}_{3}-\mathrm{SiO}_{2}$ aerogel/mullite fiber composites. Journal of Inorganic Materials, 2018, 33(9): 970-975.

[59] TANG G H, BI C, ZHAO Y, et al. Thermal transport in nano-porous insulation of aerogel: factors, models and outlook. Energy, 2015, 90: 701-721.

[60] LEE S C, CUNNINGTON G R. Conduction and radiation heat transfer in high-porosity fiber thermal insulation. Journal of Thermophysics and Heat Transfer, 2000, 14(2): 121-136.

[61] ZHAO J, DUAN Y, WANG X, et al. Radiative properties and heat transfer characteristics of fiber-loaded silica aerogel composites for thermal insulation. International Journal of Heat and Mass Transfer, 2012, 55: 5196-5204.

[62] ZHANG H, FANG W, LI Z, et al. The influence of gaseous heat conduction to the effective thermal conductivity of nano-porous materials. International Communications in Heat and Mass Transfer, 2015, 68: 158-161.

[63] 高庆福，张长瑞，冯坚，等. 氧化铝气凝胶复合材料的制备与隔 热性能. 国防科技大学学报, 2008, 30(4): 39-42.

[64] YANG G, JIANG Y, FENG J, et al. Synthesis of fibre reinforced $\mathrm{Al}_{2} \mathrm{O}_{3}-\mathrm{SiO}_{2}$ aerogel composite with high density uniformity via a facile high-pressure impregnation approach. Processing and Application of Ceramics, 2017, 11(3): 185-190.

[65] WANG K Y, LIU R X, ZHANG L, et al. Preparation and thermal stability of quartz fiber reinforced silicon doped aluminum aerogel composites. IOP Conf. Series: Materials Science and Engineering, 2019, 678: 012076

[66] ZHONG Y, ZHANG J, WU X, et al. Carbon-fiber felt reinforced carbon/alumina aerogel composite fabricated with high strength and low thermal conductivity. Journal of Sol-Gel Science and Technology, 2017, 84: 129-134.

[67] LI H, CHEN Y, WANG P, et al. Porous carbon-bonded carbon fiber composites impregnated with $\mathrm{SiO}_{2}-\mathrm{Al}_{2} \mathrm{O}_{3}$ aerogel with enhanced thermal insulation and mechanical properties. Ceramics International, 2018, 44(3): 3484-3487.

[68] 周洁洁, 王钦, 姚先周, 等. 纤维毡增强 $\mathrm{Al}_{2} \mathrm{O}_{3}-\mathrm{SiO}_{2}$ 气凝胶复合 材料的制备与隔热性能研究. 第一届中国国际复合材料科技大 会论文集, 北京, 2013: 1090-1093.

[69] XU L, JIANG Y, FENG J, et al. Infrared-opacified $\mathrm{Al}_{2} \mathrm{O}_{3}-\mathrm{SiO}_{2}$ aerogel composites reinforced by SiC-coated mullite fibers for thermal insulations. Ceramics International, 2015, 41(1): 437-442.

[70] GAO M, LIU B, ZHAO P, et al. Mechanical strengths and thermal properties of titania-doped alumina aerogels and the application as high-temperature thermal insulator. Journal of Sol-Gel Science and Technology, 2019, 91: 514-522.

[71] 隗小庆, 倪星元, 沈军, 等. 氧化铝气凝胶隔热材料的制备和热 学性能. 材料研究学报, 2012, 26(3): 261-266.

[72] 吴宇, 沈军, 祖国庆, 等. 耐高温 $\mathrm{Al}_{2} \mathrm{O}_{3}$ 基气凝胶的制备和特性 研究. 南京工业大学学报(自然科学版), 2016, 38(2): 15-19.

[73] ZOU W, WANG X, WU Y, et al. Highly thermally stable aluminabased aerogels modified by partially hydrolyzed aluminum tri-sec-butoxide. Journal of Sol-Gel Science and Technology, 2017, 84: 507-514.

[74] 王虹, 周裴灿, 李荣年, 等. 氧化硅气凝胶与氧化铝气凝胶的性 能对比. 中国建材科技, 2019(6): 34-35.

[75] 冯坚. 气凝胶高效隔热材料. 北京: 科学出版社, 2016: 1-206. 